\title{
Effect of Pilates exercises on postpartum maternal fatigue
}

\author{
Farzaneh Ashrafinia ${ }^{1}$, Msc, Mandana Mirmohammadali ${ }^{2}$, MSc, Hamid Rajabi $^{3}$, PhD, Anoshirvan Kazemnejad ${ }^{4}$, PhD, \\ Khosro Sadeghniiat Haghighi ${ }^{5}$, MD, Mehrnoosh $\underline{\text { melvalizadeh }}^{6}$, MSc
}

INTRODUCTION Postpartum fatigue is a pervasive phenomenon and often affects mothers immediately after delivery. The present study aimed to assess the effect Pilates home exercises had on postpartum maternal fatigue.

METHODS A total of 80 women participated in our clinical trial study. The women were randomly divided into two groups - the intervention group $(n=40)$ and the control group $(n=40)$. In the intervention group, the women performed Pilates exercises five times a week (30 min per session) for eight consecutive weeks. The first session was conducted 72 hours after delivery. The control group did not receive any intervention. Each woman's level of fatigue was evaluated at hospital discharge (as a baseline), and at four and eight weeks after delivery, using the standard Multidimensional Fatigue Inventory (MFI-20) questionnaire and repeated measures analysis.

RESULTS During the eight weeks of follow-up, we found that the intervention group had lower mean MFI-20 scores than the control group with regard to general fatigue ( $7.80 \pm 2.07$ vs. $12.72 \pm 1.79 ; p<0.001)$, physical fatigue $(7.12 \pm 1.41$ vs. $10.42 \pm 2.02 ; p<0.001)$, reduced activity $(6.95 \pm 1.35$ vs. $11.27 \pm 1.70 ; p<0.001)$, reduced motivation $(6.20 \pm 1.01$ vs. $9.80 \pm 2.04 ; p<0.001)$ and mental fatigue $(6.85 \pm 1.45$ vs. $10.72 \pm 1.98 ; p<0.001)$.

CONCLUSION The present study's findings show that physical exercise can significantly reduce postpartum maternal fatigue in all subscales.

Keywords: fatigue, Pilates, postpartum period

\section{INTRODUCTION}

The postpartum period is a transitional phase that has a decisive effect on a mother's physical and mental health. ${ }^{(1)}$ The changes involved during this period, including hormonal shifts and the beginning of new and unfamiliar child-rearing tasks, can cause primiparous mothers to experience stress and fatigue. ${ }^{(2)}$

Postpartum fatigue, which is the result of the tense and overwhelming experience of caring for a newborn, has negative effects on the health of the mother, the neonate and the family. ${ }^{(3)}$ This phenomenon often begins immediately after delivery and reaches its maximum severity within 36 hours. ${ }^{(4)}$ In contrast to the belief that it is a temporary experience, postpartum fatigue can last for a prolonged period after delivery. ${ }^{(3)} \mathrm{A}$ literature review by Groër et al reported that more than $80 \%$ of mothers complain of postpartum fatigue. ${ }^{(5)}$ Corwin et al reported a postpartum fatigue incidence of about $70 \%$ among mothers who had given birth 1-2 months earlier. ${ }^{(6)}$ Primiparous women were found to be more likely to complain of postpartum fatigue. ${ }^{(7)}$

Although postpartum fatigue is a common and natural phenomenon, it has received attention due to its excruciating nature. ${ }^{(8)}$ Even after more than two decades of study, postpartum fatigue remains one of the five major postpartum concerns and its treatment is often a controversial subject in many studies. ${ }^{(9)}$ The resultant reduced stamina decreases a mother's capability to undertake physical and mental tasks; it also decreases her ability to manage her infant's needs, her responsibilities to other family members and her professional duties. ${ }^{(10)}$ Additionally, reduced maternal energy impairs the immune, nervous and mental systems of both the mother and the infant. ${ }^{(5)}$ Other undesirable and potentially serious outcomes include termination of lactation, (11) impairment of the sexual relationship between spouses, ${ }^{(7)}$ development of stress and mood disorders ${ }^{(5)}$ and an increased risk of postpartum depression. ${ }^{(12)}$ Therefore, it is of utmost importance that an effective solution to this condition is found. There has been some evidence supporting the positive effect of physical exercise on maternal and infant health. ${ }^{(1)}$ The theory behind the use of physical exercise is that, by increasing maternal motivation and self-confidence, postpartum exercise enhances child-rearing practices and helps mothers play their maternal roles more efficiently. ${ }^{(13)}$

Considering the circumstances of a postpartum woman, home exercise is deemed the most suitable and is also the most common type of exercise recommended by healthcare professionals, since it presents fewer childcare-related barriers, resulting in mothers exercising more willingly and with greater motivation. ${ }^{(14)}$ Pilates exercises, introduced by Joseph Pilates in the early 20th century, are considered a good method for accelerating convalescence after pregnancy. However, the effectiveness of Pilates exercises in reducing postpartum fatigue is not well known. ${ }^{(15)}$ In a previous study, ${ }^{(1)}$ the combination of yoga and Pilates exercises was shown to result in a significant reduction of postpartum depression and fatigue. In that study, however, the effect of Pilates exercises alone was not quantified. Therefore, the aim of the present study was to assess the effect of a set of Pilates home exercises on the general, physical and mental fatigue of mothers, as well as on the activity and motivation levels of the mothers.

${ }^{1}$ Department of Midwifery, School of Nursing and Midwifery, Kerman University of Medical Sciences, Kerman, ${ }^{2}$ Department of Reproductive Health, School of Nursing and Midwifery, Tehran University of Medical Sciences, ${ }^{3}$ Department of Sport Physiology, Tarbiat Moallem University, ${ }^{4}$ Department of Biostatistics, Tarbiat Modares University, ${ }^{5}$ Department of Occupational Medicine, Tehran University of Medical Sciences, ${ }^{6}$ Department of Reproductive Health, School of Nursing and Midwifery, Tehran University of Medical Sciences, Tehran, Iran

Correspondence: Farzaneh Ashrafinia, Instructor, Department of Midwifery, School of Nursing and Midwifery, Kerman University of Medical Sciences, Haft Bagh Blvd, Keman 7616913555, Iran. Ashrafifarzaneh60@yahoo.com 


\section{METHODS}

The present study was a randomised clinical trial that aimed to evaluate the effect of Pilates exercises on postpartum maternal fatigue. Primiparous women who attended any one of the seven Rafsanjan Health Centres in Iran for prenatal care were eligible for inclusion in the present study. In order to be included, primiparous women also had to meet the following criteria: (a) possess Iranian nationality; (b) aged 18-35 years; (c) singleton pregnancy; and (d) no history of physical and mental diseases. The women were enrolled in our study after normal vaginal delivery of a healthy term neonate. Individuals were excluded if they had any of the following: (a) postpartum depression score $\geq$ 10, based on the Edinburgh Postnatal Depression Scale (EPDS); ${ }^{(16)}$ (b) postpartum anaemia; and (c) respiratory infection. Individuals who had emigrated, missed three successive exercise sessions or had five interrupted sessions (as reported in the weekly checklist), or had hospitalisation of their neonate for any reason were also excluded from the study. Participants were selected from the aforementioned health centres using randomised blocking. Four health centres were randomly selected out of the seven, and individuals who attended those four centres were assigned to the intervention group. Individuals who attended the remaining three centres were assigned to the control group. Data was collected from March 2009 through September 2009.

Given that $\alpha<0.05$ and $\beta=0.2$ (i.e. the standard values, which allow for $80 \%$ power, in sample size calculations), 33 participants were required for each group, based on an estimated $20 \%$ probability of loss to follow-up during the study. We recruited a total of 40 women for each group (inclusion in the study was based on the aforementioned criteria). All participants $(\mathrm{n}=80)$ gave written informed consent to participate in the study. Since the intervention could not be masked, the present study was performed as an open-label clinical trial. None of the women in the intervention and control groups had postpartum depression (according to the EPDS scores) and none had to be excluded due to postpartum anaemia. In addition, none of the women were reported to have postpartum infection (i.e. metritis, mastitis, wound infection or urinary infection). The present study was approved by the ethics committee of Tehran University of Medical Sciences (No. 10301) and the Iranian Registry of Clinical Trials (Registration no. IRCT 201102275912N2).

In the present study, intervention comprised a schedule of Pilates home exercises that were suitable for women during the postpartum period. The Pilates home exercises consisted of a set of 13 movements: bridging; hundred; roll up; one leg circle (both ways); rocker with closed legs; single straight leg stretch; double leg stretch; spine stretch forward; single leg kick; side kick up and down; side kick circles; rest position (stretch and relaxation); and curling. These 13 movements were derived from the following books: Women's Health and Fitness Guide ${ }^{(14)}$ and Fitness Professional's Handbook. ${ }^{(15)}$ The women were instructed to perform 3-5 repetitions of the 13 movements at the start, and to add two more repetitions for each movement every week until the end of the intervention.

The exercises were stretching movements that focused on deep breathing, and included whole-body stretching and core strengthening. The suitability of these exercises for women during the postpartum period was approved by a sports physiologist. The assigned exercises were started 72 hours after delivery, and as long as there was no obstetric or medical problem to prevent the women from performing the exercises, they were continued for eight weeks. In the event of a medical problem, the first exercise period could be delayed until the end of the first week. The exercises were performed five days a week (preferably on successive days), for half an hour each day, in accordance with the recommendations of the American College of Obstetricians and Gynecologists. ${ }^{(14)}$ Preferably, the exercises were to be done in the morning, after the infants were fed. The number of repetitions and the intensity of each exercise were gradually increased throughout the duration of the intervention.

To ensure that the exercises were performed correctly, the women in the intervention group received four training sessions over the course of the three weeks prior to delivery. The training consisted of video screenings of the Pilates home exercises to be performed during the postpartum period as well as explanations on postpartum exercises. The women in the intervention group were given a video, a training booklet and an audio CD to use during home exercise. In order to ensure correct implementation of the intervention protocol, a researcher established good relationships with the participants and made weekly phone calls. The researcher also visited each participant every two weeks to review the exercises and to answer the participant's questions. This was done to ensure that the exercises were being performed correctly. In addition, participants were given a diary to record their exercise sessions. This diary was reviewed by the researcher at each visit.

One training session on postpartum care was held for the control group in order to motivate them to participate in the present study. The women in this group were asked to report any extra physical activity performed during the study period. They were also monitored via telephone calls during the duration of the study.

In the present study, fatigue was measured using the standard Multidimensional Fatigue Inventory (MFI-20) questionnaire. ${ }^{(17)}$ This 20-item questionnaire measures the severity of five subsets of fatigue - general fatigue, physical fatigue, mental fatigue, reduced activity and reduced motivation. Each subset is assessed by four items of the questionnaire. Higher scores in a subset represent a greater degree of that type of fatigue. The validity and reliability of this questionnaire for measuring fatigue during pregnancy and after delivery have been verified. ${ }^{(18)}$ In the present study, the validity of the MFI-20 questionnaire after translation was approved by experts. The translated MFI-20 questionnaire had a Cronbach's alpha of $77.3 \%$, indicating its reliability for use in populations similar to our study population. The MFI-20 questionnaire was filled out by a researcher at a health centre at the time of hospital discharge (i.e. 24 hours after delivery), and at the end of the first and second four-week period (i.e. end of the fourth and eighth week) after delivery. If a pregnant woman from our study cohort was not admitted to a hospital for delivery, the researcher filled out the questionnaire upon visiting her at her house. 
Considering the effect of depression on the incidence and severity of fatigue, all participants in the present study were assessed for depression using the EPDS. Women who were found to be depressed (i.e. EPDS score $\geq 10$ ) were excluded. EPDS, which comprises ten items divided into four subsets, is an advanced and valid scale for evaluating postpartum depression. These items evaluate maternal mood disorders, but do not consider physical symptoms. ${ }^{(19)}$ Montazeri et al studied the content validity of the Persian version of the EPDS and reported an r-value of 0.77 for its test-retest reliability. ${ }^{(20)}$ Since there is documented evidence that anaemia can result in fatigue, ${ }^{(12)}$ a complete blood count was performed four weeks after delivery for all the women in both groups to diagnose postpartum anaemia. Other information, including the demographics of the participants, was collected at the time of enrollment into the present study. All of the aforementioned questionnaires were completed via interviews with the participants.

Data analysis was performed using the Statistical Package for the Social Sciences version 16 (SPSS Inc, Chicago, IL, USA). Descriptive statistics, $t$-tests and chi-square tests were applied to assess baseline demographic variables and to compare these variables between the intervention and control groups. The effect Pilates home exercises had on postpartum maternal fatigue was examined by measuring mean changes in the fatigue scores of both sample groups over time, using repeated measures analysis (i.e. repeated analysis of variance between the intervention and control groups).

\section{RESULTS}

There was no significant difference observed between the intervention group $(n=40)$ and the control group $(n=40)$ in terms of age, educational level and occupational status (Table I). There was also no significant difference between the two groups in terms of the age, educational level and occupational status of the women's husbands.

The two sample groups showed no significant postpartum difference for any of the following potentially confounding factors: (a) consumption of tea, coffee or herbal drugs; (b) experience of pain; (c) rate of physical activity; (d) presence of help with household tasks; (e) presence of help with child-rearing; and (f) overall infant sleep status $(p>0.3)$ (results not shown). Matched results of the repeated measures analysis done revealed no significant difference between the two groups with regard to the severity of fatigue 24 hours after delivery. However, at both four weeks and eight weeks after the initiation of the Pilates home exercises in the intervention group, there were significant differences in the severity of fatigue between the intervention and control groups. When compared with the control group, the intervention group was found to have significant improvement in the areas of general fatigue $(p<0.001)$, physical fatigue $(p<0.001)$, reduced activity $(p<0.001)$, reduced motivation $(p<0.001)$ and mental fatigue $(p<0.001)$ (Table II).

\section{DISCUSSION}

The present study is one of the few studies that investigated the effect of Pilates exercises on the severity of postpartum maternal
Table I. Demographic characteristics of the women in the control $(n=40)$ and intervention $(n=40)$ groups.

\begin{tabular}{|c|c|c|c|}
\hline \multirow[t]{2}{*}{ Variable } & \multicolumn{2}{|c|}{ No. of women (\%) } & \multirow[t]{2}{*}{ p-value $^{+}$} \\
\hline & Control & Intervention & \\
\hline Age* $(y r)$ & $24.4 \pm 3.6$ & $24.6 \pm 3.6$ & 0.91 \\
\hline Husband's age* (yr) & $27.6 \pm 3.4$ & $29.3 \pm 3.8$ & 0.09 \\
\hline \multicolumn{4}{|l|}{ Highest education } \\
\hline $\begin{array}{l}\text { Lower than high } \\
\text { school diploma }\end{array}$ & $13(32.5)$ & $4(10.0)$ & 0.24 \\
\hline Diploma & $16(40.0)$ & $24(60.0)$ & \\
\hline University & $11(27.5)$ & $12(30.0)$ & \\
\hline \multicolumn{4}{|l|}{ Occupation } \\
\hline Unemployed & $35(87.5)$ & $32(80.0)$ & 0.36 \\
\hline Employed & $5(12.5)$ & $8(20.0)$ & \\
\hline \multicolumn{4}{|l|}{$\begin{array}{l}\text { Husband's highest } \\
\text { education }\end{array}$} \\
\hline $\begin{array}{l}\text { Lower than high } \\
\text { school diploma }\end{array}$ & $23(57.5)$ & $13(32.5)$ & 0.16 \\
\hline Diploma & $13(32.5)$ & $16(40.0)$ & \\
\hline University & $4(10.0)$ & $11(27.5)$ & \\
\hline \multicolumn{4}{|l|}{ Husband's occupation } \\
\hline Factory labourer & $6(15.0)$ & $12(30.0)$ & 0.26 \\
\hline Civil servant & $3(7.5)$ & $3(7.5)$ & \\
\hline Self-employed & $31(77.5)$ & $25(62.5)$ & \\
\hline
\end{tabular}

* Data is presented as mean \pm standard deviation. ${ }^{t} \mathrm{p}$-value $<0.05$ is considered statistically significant.

fatigue. ${ }^{(1,21,22)}$ In our study, we found that Pilates home exercises were able to reduce postpartum maternal fatigue. The exercises were shown to reduce general, physical and mental fatigue, and improve activity and motivation. In fact, in each of these subsets, major improvements were associated with the practice of Pilates home exercises.

According to available evidence, ${ }^{(1,19)}$ postpartum maternal fatigue is generally characterised as a dynamic, complicated and multidimensional phenomenon that negatively affects a mother's mental, physical and social health. Manifestations of postpartum fatigue (e.g. hesitation, persistent concern, impatience, mixed thoughts, and decreased concentration and memory performance) are potentially painful for women and can put them in a vicious cycle that worsens the fatigue. As the symptoms of postpartum fatigue often affect the mental state of the women, these symptoms may not be readily observable to family members. To support these women, there is a need to develop effective interventions for postpartum fatigue. ${ }^{(23)}$

Studies on Pilates exercises have indicated both mental and physical effects on health. ${ }^{(24)}$ For many years, Pilates exercises have been regarded as a useful tool for rehabilitation. ${ }^{(22)}$ In 2008, Keays et al reported the beneficial effect of Pilates exercises on temperament and depression. ${ }^{(25)}$ Findings of a study conducted by Eyigor et al showed that Pilates exercises were a healthy and effective method for improving the functional capacity, flexibility, fatigue, depression and quality of life of breast cancer patients. ${ }^{(22)}$ In a randomised clinical trial, Ko et al reported that the combination of Pilates and yoga exercises had a positive effect on the fatigue and depression symptoms of postpartum women; the effects of the 
Table II. Effect of the Pilates home exercises intervention, as measured by the Multidimensional Fatigue Inventory questionnaire.

\begin{tabular}{|c|c|c|c|c|c|c|}
\hline \multirow[t]{2}{*}{ Variable } & \multicolumn{3}{|c|}{ Mean \pm standard deviation } & \multicolumn{3}{|c|}{ p-value* } \\
\hline & At discharge & 4 weeks postpartum & 8 weeks postpartum & Group effect & Time effect & Overall effect \\
\hline \multicolumn{7}{|l|}{ General fatigue } \\
\hline Intervention group & $11.15 \pm 2.17$ & $9.12 \pm 2.02$ & $7.80 \pm 2.07$ & $<0.001$ & $\leq 0.010$ & $<0.001$ \\
\hline Control group & $11.52 \pm 2.40$ & $11.67 \pm 2.84$ & $12.72 \pm 1.79$ & & & \\
\hline \multicolumn{7}{|l|}{ Physical fatigue } \\
\hline Control group & $12.35 \pm 1.87$ & $10.82 \pm 2.29$ & $10.42 \pm 2.02$ & & & \\
\hline \multicolumn{7}{|l|}{ Reduced activity } \\
\hline Intervention group & $12.02 \pm 1.84$ & $7.87 \pm 1.53$ & $6.95 \pm 1.35$ & $<0.001$ & $<0.001$ & $<0.001$ \\
\hline Control group & $12.80 \pm 1.82$ & $11.02 \pm 2.33$ & $11.27 \pm 1.70$ & & & \\
\hline \multicolumn{7}{|l|}{ Reduced motivation } \\
\hline Intervention group & $7.92 \pm 1.14$ & $6.77 \pm 1.40$ & $6.20 \pm 1.01$ & $<0.001$ & 0.145 & $<0.001$ \\
\hline Control group & $8.50 \pm 1.43$ & $8.85 \pm 1.79$ & $9.80 \pm 2.04$ & & & \\
\hline \multicolumn{7}{|l|}{ Mental fatigue } \\
\hline Intervention group & $8.82 \pm 1.75$ & $7.25 \pm 1.67$ & $6.85 \pm 1.45$ & $<0.001$ & 0.446 & $<0.001$ \\
\hline Control group & $9.35 \pm 2.11$ & $10.42 \pm 2.27$ & $10.72 \pm 1.98$ & & & \\
\hline
\end{tabular}

$*_{p}$-value $<0.05$ is considered statistically significant

exercises were measured using the Fatigue Symptom Checklist. ${ }^{(1)}$ In a study by Drista et al, which evaluated the effect of aerobic home exercises on fatigue in women with postpartum depression, the authors found a significant reduction in all subsets of fatigue, except mental fatigue, when maternal fatigue was measured using the MFI-20 questionnaire. ${ }^{(19)}$ Therefore, the ability of physical exercise to successfully and significantly decrease patient fatigue is evident in past studies. The agreement of our findings with that of previous studies clearly indicates the strong effect exercise has on reducing fatigue. According to these studies, the mechanism by which physical exercise positively affects postpartum fatigue is likely similar to the mechanism by which physical exercise positively affects chronic diseases. ${ }^{(26-28)}$

In the present study, the Pilates home exercises did not result in any adverse effects or undesirable outcomes for the mothers. Moreover, the nature (i.e. low-impact, non-aerobic and deepmuscle conditioning) and the purposeful design of the exercises made them practical and enjoyable for the mothers. The positive physical effects of these exercises, which include strengthened core muscles (i.e. abdominal, gluteal and dorsal muscles) and improved flexibility and stability, as well as the positive mental effects of these exercises, make them a useful and practical means for recovering and improving one's quality of life. ${ }^{(15)}$ Home exercises result in changes that can help mothers more easily perform the physical and manual tasks involved in child-rearing; home exercises may also increase the mother's ability to take their children out of the house. ${ }^{(19)}$

To the best of our knowledge, no study has yet been conducted to assess the effect of Pilates exercises alone on postpartum maternal fatigue. One of the strengths of the present study is that it extensively evaluated the benefits of Pilates home exercises with regard to various subsets of fatigue in healthy women during the postpartum period. Maternal fatigue is strongly associated with the incidence of postpartum depression, affecting the quality of life of the mother and her family. Therefore, relieving or reducing the severity of postpartum fatigue and devising helpful interventions for this purpose is crucial. ${ }^{(3)}$

The present study was not without limitations. As it was conducted on primiparous women, caution should be exercised if our findings are to be extrapolated to multiparous women. The biological status, adaptive power, skills and self-efficacy of multiparous women are different from those of primiparous women, ${ }^{(14)}$ and may cause them to have different responses to intervention. In the present study, there was also the possibility of recall bias or incorrect reporting due to the self-reported nature of the data. We attempted to decrease this via the use of direct communication with the participants (to build their confidence and motivation), regular reminders on the importance of the study and timely follow-up sessions. Another limitation was the short timeline over which the Pilates home exercise regimen was performed. Further studies with larger sample sizes, and longer intervention and follow-up periods are recommended in order to compare different exercise protocols. For studies in which exercises are performed over a longer period of time, the exercises performed must be adapted over time, according to the changes in the physiological and physical statuses of the mothers.

In conclusion, the results of the present study show that Pilates home exercises are an effective, healthy and feasible method for reducing postpartum fatigue. Pilates exercises are popular because they are economical, can be performed at home and do not interfere with child-rearing. Reducing fatigue, which decreases the risk of depression, is an effective step in promoting the health of mothers and infants. Therefore, more attention should be paid to effective interventions, such as physical exercises, for reducing fatigue.

\section{ACKNOWLEDGEMENTS}

We wish to thank Tehran University of Medical Sciences, Iran, for its financial support and cooperation throughout the course 
of the study, as well as the mothers who had voluntarily taken part in this study.

\section{REFERENCES}

1. Ko YL, Yang CL, Chiang LC. Effects of postpartum exercise program on fatigue and depression during "doing-the-month" period. J Nurs Res 2008; 16:177-86.

2. Heh SS, Huang LH, Ho SM, Fu YY, Wang LL. Effectiveness of an exercise support program in reducing the severity of postnatal depression in Taiwanese women. Birth 2008; 35:60-5.

3. Dennis $\mathrm{CL}$, Ross L. Relationships among infant sleep patterns, maternal fatigue, and development of depressive symptomatology. Birth 2005; 32:187-93.

4. Lee SH. Effects of aroma inhalation on fatigue and sleep quality of postpartum mothers. Korean J Women Health Nurs 2004; 10:235-42.

5. Groër M, Davis M, Casey $K$, et al. Neuroendocrine and immune relationships in postpartum fatigue. MCN Am J Matern Child Nurs 2005; 30:133-8.

6. Corwin EJ, Brownstead J, Barton N, Heckard S, Morin K. The impact of fatigue on the development of postpartum depression. J Obstet Gynecol Neonatal Nurs 2005; 34:577-86.

7. Troy NW. Is the significance of postpartum fatigue being overlooked in the lives of women? MCN Am J Matern Child Nurs 2003; 28:252-7.

8. Doering JJ, Morin K, Stetzer FC. Severe fatigue and depressive symptoms in lower-income urban postpartum women. West J Nurs Res 2009; 31:599-612.

9. Taylor J, Johnson M. How women manage fatigue after childbirth. Midwifery 2010; 26:367-75.

10. Rychnovsky JD. Postpartum fatigue in the active-duty military woman. J Obstet Gynecol Neonatal Nurs 2007; 36:38-46.

11. Callahan S, Séjourné N, Denis A. Fatigue and breastfeeding: an inevitable partnership? J Hum Lact 2006; 22:182-7.

12. Corwin EJ, Arbour M. Postpartum fatigue and evidence-based interventions. MCN Am J Matern Child Nurse 2007; 32:215-20.

13. Østbye T, Krause KM, Lovelady CA, et al. Active Mothers Postpartum: a randomized controlled weight-loss intervention trial. Am J Prev Med 2009; 37:173-80.

14. Kettles MA, Wright BS, Cole CL. Women's Health and Fitness Guide. 1st ed. Human Kinetics, 2006: 77-104

15. Howley ET, Franks BD. Fitness Professional's Handbook. 15th ed. Human Kinetics, 2007: 351-60.

16. Cox JL, Holden JM, Sagovsky R. Detection of postnatal depression: Development of the 10-item Edinburgh postnatal depression scale. Br J Psychiatry 1987;150:782-786.

17. Smets EM, Garssen B, Bonke B, de Haes JC. The Multidimensional Fatigue Inventory (MFI) psychometrics qualities of an instrument to assess fatigue. J Psychosom Res 1995; 39:315-25.

18. Fairbrother N, Hutton EK, Stoll K, Hall W, Kluka S. Psychometric evaluation of the Multidimensional Assessment of Fatigue scale for use with pregnant and postpartum women. Psychol Assess 2008; 20:150-8.

19. Drista M, Da Costa D, Dupuis G, Lowensteyn I, Khalifé S. Effects of a home-based exercise intervention on fatigue in postpartum depressed women: results of a randomized controlled trial. Ann Behav Med 2008; 35:179-87.

20. Montazeri A, Torkan B, Omidvari S. The Edinburgh Postnatal Depression Scale (EPDS): translation and validation study of the Iranian version. BMC Psychiatry 2007; 7:11.

21. Ko YL, Yang CL, Fang CL, Lee MY, Lin PC. Community-based postpartum exercise program. J Clin Nurs 2013; 22:2122-31.

22. Eyigor S, Karapolat H, Yesil H, Uslu R, Durmaz B. Effects of pilates exercises on functional capacity, flexibility, fatigue, depression and quality of life in female breast cancer patients: a randomized controlled study. Eur J Phys Rehabil Med 2010; 46:481-7.

23. Runquist J. Persevering through postpartum fatigue. J Obstet Gynecol Neonatal Nurs 2007; 36:28-37.

24. Bernardo LM. The effectiveness of Pilates training in healthy adults: An appraisal of the research literature. J Bodyw Mov Ther 2007; 11:106-10.

25. Keays KS, Harris SR, Lucyshyn JM, Maclntyre DL. Effects of Pilates exercises on shoulder range of motion, pain, mood, and upper-extremity function in women living with breast cancer: a pilot study. Phys Ther 2008; 88:494-510.

26. Miller RG. Fatigue and therapeutic exercise. J Neurol Sci 2006; 242:37-41.

27. Mock V, Frangakis C, Davidson NE, et al. Exercise manages fatigue during breast cancer treatment: a randomized controlled trial. Psychooncology 2005; 14:464-77.

28. Oken BS, Kishiyama S, Zajdel D, et al. Randomized controlled trial of yoga and exercise in multiple sclerosis. Neurology 2004; 62:2058-64. 